ARTIGO ORIGINAL

ISSN 1677-5090

(C) 2019 Revista de Ciências Médicas e Biológicas

http://dx.doi.org/10.9771/cmbio.v18i3.34187

\title{
Achados da biomecânica de deglutição em pacientes com megaesôfago chagásico
}

\author{
Findings of the Swallowing Biomechanics in Patients with Chagasic Megaesophagus
}

Ana Catarina Moura Torres ${ }^{1}$, Jorge de Carvalho Guedes², Ana Caline Nóbrega da Costa ${ }^{3 *}$

${ }^{1}$ Mestre em Alimentos, nutrição e saúde, UFBA.; ${ }^{2}$ Doutor em Medicina, UFBA.;

${ }^{3}$ Doutora em Medicina e Saúde, UFBA.

\begin{abstract}
Resumo
O megaesôfago chagásico é uma alteração do trato gastrointestinal caracterizada pela destruição ou ausência de plexos nervosos intramurais do esôfago. Alterações nutricionais e da saúde pulmonar são descritas como consequentes a esse diagnóstico. Objetivo: descrever os achados das fases oral e faríngea da deglutição em indivíduos portadores de Megaesôfago Chagásico Metodologia: participaram desta pesquisa 26 voluntários com diagnóstico confirmado de doença de Chagas. Todos realizaram avaliação videofluoroscópica da deglutição, ao deglutir sólido, líquido, semilíquido e pastoso. Resultados: observou-se prevalência do sexo feminino $(73,1 \%)$, e os graus I e II de classificação do megaesôfago foram os mais encontrados. A ausência de unidades dentárias posteriores esteve presente em mais de $90 \%$ na população e o tipo de mastigação anterior foi observado em $40,7 \%$ da amostra. Durante o exame videofluoroscópico, a presença de resíduo oral, faríngeo e em região de transição faringoesofágica foi mais encontrado na consistência semilíquida, além de um tempo de trânsito oral total aumentado para o sólido. Conclusão: escape posterior, resíduos orais, faríngeos e na transição faringoesofágica na consistência semi-líquida foram os achados mais encontrados no grupo estudado. Palavras-chaves: Deglutição. Doença de Chagas. Disfagia.
\end{abstract}

\begin{abstract}
Chagasic Megaesophagus is an alteration of the gastrointestinal tract, characterized by the destruction or absence of intramural nerve plexuses of the esophagus. Nutritional and pulmonary health changes are described as a consequence of this diagnosis. Objective: to describe the findings of the oral and pharyngeal stages of swallowing in individuals with Chagasic Megaesophagus. Methodology: twenty - six volunteers with confirmed diagnosis of Chagas' disease participated in this research. All performed videofluoroscopic swallowing evaluation when swallowing solid, liquid, semi-liquid and pasty food. Results: there was a prevalence of females (73.1\%), and megaesophagus grades I and II were found most often. The absence of posterior dental units was present in more than $90 \%$ in the population and the anterior chewing type was observed in $40.7 \%$ of the sample. During videofluoroscopic examination, the presence of oral, pharyngeal and pharyngoesophageal transition region residues was found more in semi-liquid consistency in addition to having an increased total oral transit time for the solid ones. Conclusion: the presence of posterior escape, oral, pharyngeal and pharyngoesophageal transition residues in semi-liquid consistency was found.
\end{abstract}

Keywords: Swallowing. Chagas' disease. Dysphagia

\section{INTRODUÇÃO}

A doença de Chagas é caracterizada pelo acometimento de tecidos de humanos e animais a partir da transmissão pelo protozoário Trypanossoma Cruzy (BRASIL. MINISTÉRIO DA SAÚDE, 2013). Inicialmente descrita em 1909, pelo brasileiro Carlos Chagas, essa doença acomete cerca de 20 milhões de pessoas nos países da América, sendo também descrita em países como a Austrália, Japão, Canadá e França (MURILLO-GODÍNEZ, 2018).

A transmissão ocorre a partir da deposição de fezes contaminadas do vetor em peles lesadas, de transfusões sanguíneas, é congênita,ou seja,transmitida de mães infectadas para seus filhos, também por transplantes de

Correspondente/Corresponding: *Ana Caline Nóbrega da Costa Instituto de Ciência da Saúde, Universidade Federal da Bahia, Departamento de Fonoaudiologia - End: Av. Reitor Miguel Calmon, s/n, Canela Salvador/BA. - Tel: (71) 99975-9905-E-mail: anacalinen@gmail.com órgãos, acidentes de laboratórios e pela transmissão oral (BRASIL. MINISTÉRIO DA SAÚDE, 2013). Alguns estudos descrevem a transmissão por ingestão de alimentos contaminados, como açaí, caldo de cana e carne crua advinda de animais infectados (MURILLO-GODÍNEZ, 2018; SANGENIS et al, 2016; SANTOS et al, 2019).

$\mathrm{Na}$ fase aguda da doença, febre, mialgia, cefaleia e edema são observados no período da incubação enquanto que alterações cardíacas e digestivas, principalmente esôfago e cólon, são descritos na fase crônica (DIAS et al., 2016).

A alteração mais comum no esôfago é o megaesôfago chagásico (BUENO; ALVES, 2015), caracterizado pela desnervação intrínseca do esôfago, ocasiona perda do peristaltismo no corpo do esôfago e comprometimento do relaxamento do esfíncter esofagiano inferior (REZENDE, 1997). Essas alterações são secundárias a 
lesões degenerativas do plexo mientérico de Auerbach (REZENDE, 1997).

Estudos (SANTOS; CASSIANI; DANTAS, 2012) descrevem alterações na biomecânica da deglutição de indivíduos portadores de doença de Chagas, como, por exemplo, aumento do tempo de trânsito oral e faríngeo, depuração faríngea mais longa, maior frequência de deglutições múltiplas e elevação laríngea reduzida em relação a sujeitos saudáveis na deglutição de alimentos com consistências líquida e pastosa.

Conhecer a biomecânica de deglutição de indivíduos portadores de megaesôfago chagásico é essencial para direcionar ações no sentido de reduzir riscos relacionados à alimentação dessa população, assim como para tornar o processo alimentar mais funcional. Desta forma, o objetivo deste estudo é descrever os achados das fases oral e faríngea da deglutição em indivíduos portadores de Megaesôfago Chagásico.

\section{METODOLOGIA}

Trata-se de um estudo de corte transversal e descritivo, onde foram utilizadas informações de prontuários e resultados de exame videofluoroscópico da deglutição, de uma amostra de conveniência, composto por indivíduos que compareceram ao ambulatório de Gastroenterologia do Complexo Universitário HUPES, no período de agosto/2010 a dezembro/2010.

\section{Critérios de Inclusão}

Foram incluídos no estudo os pacientes adultos e idosos com diagnóstico confirmado de megaesôfago chagásico estabelecido por meio dos seguintes critérios:

- Clínicos: Presença de disfagia progressiva com duração superior a um ano, com evidências sorológicas de contato com o T cruzi;

- Radiológicos: Presença de dilatação do esôfago e/ou lentificação do trânsito;

e/ou

- Manométricos: Relaxamento incompleto do esfíncter esofageano inferior à passagem do bolo alimentar, associado ou não à hipomotilidade do corpo esofágico.

O protocolo de estudo foi aprovado pelo Comitê de Ética em Pesquisa do Complexo Universitário Professor Edgard Santos, UFBA em 28/10/2010, cadastro 20/10. Todos os participantes concordaram em assinar o Termo de Consentimento Livre e Esclarecido.

\section{Critérios de Não Inclusão}

Os voluntários não incluídos no estudo foram aqueles com lesões estruturais orais, faringolaríngeas e/ou esofágicas, como tumores, cicatrizes por radioterapia e ausência de estruturas. Nenhum dos voluntários foi excluído por apresentar esses critérios. Adotou-se também, como critério de não inclusão, a submissão prévia à cirurgia de ressecção esofágica.

Foi realizada a análise de dados de prontuário de todos os sujeitos da pesquisa e todos foram submetidos à avaliação videofluoroscópica da deglutição.

Os dados coletados dos prontuários foram referentes à realização de procedimentos cirúrgicos relacionados ao megaesôfago.

\section{Exame Videofluoroscópico}

Os exames foram realizados no Serviço de Radiologia do Hospital Universitário Professor Edgar Santos por um radiologista e um fonoaudiólogo.

O sulfato de bário foi utilizado como contraste. A avaliação videofluoroscópica incluiu o registro da deglutição, em posição lateral, com ingestão de alimentos contrastados em diferentes volumes e consistências, com base em Gonçalves e Lederman (2004) . O alimento sólido foi $1 / 2$ unidade de biscoito waffer. O pastoso, uma colher de sobremesa $(5 \mathrm{ml})$ e uma colher de sopa $(10 \mathrm{ml})$ de iogurte cremoso Nestlé ${ }^{\oplus}$ desnatado misturado ao bário (2:1). Para o semilíquido foi utilizado o bário puro, ofertas de uma colher de sobremesa $(5 \mathrm{ml})$ e uma colher de sopa $(10 \mathrm{ml})$ ). Para o líquido, $5 \mathrm{ml}$ e $20 \mathrm{ml}$ de bário diluído em água (1:1).

A avaliação da fase esofágica da deglutição foi realizada com o sujeito de pé, em posição ântero-posterior.

As imagens foram gravadas em mídia, por meio de um gravador de DVD, da marca Sony, acoplado ao monitor do equipamento.

\section{Definição dos Parâmetros Analisados Quantitativamente e Qualitativamente}

\section{Tempo de Trânsito Oral Total}

Tem seu início marcado como o primeiro momento do bolo na cavidade oral e o seu término quando a parte proximal do bolo alimentar atinge a hipofaringe ou quando a borda inferior da mandíbula atravessa a base da língua, conforme descrito em Gatto (2010).

O tempo de trânsito oral foi marcado pela contagem dos segundos visualizados no cronômetro do aparelho de DVD, considerando o início e o final descritos acima. 
Figura 1 - Figura ilustrando os momentos de início e término do tempo de trânsito oral total.

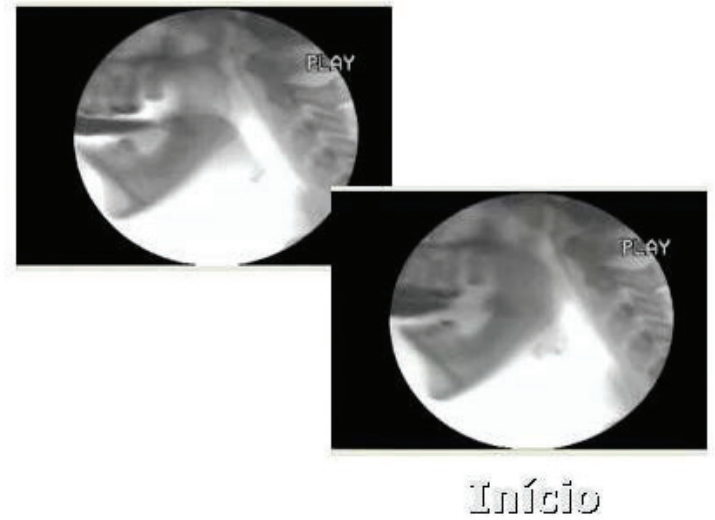

Fonte: Gatto (2010)

\section{Escape Oral Posterior}

É a posição do bolo alimentar onde se inicia a resposta faríngea. Para sua classificação foi usada uma escala baseada em Gatto (2010). O bolo alimentar em qualquer região entre pilar das fauces e o ponto onde o dorso da língua cruza a borda inferior da mandíbula.

1. Bolo alimentar no dorso da língua quase atingindo a valécula.

2. 2,5 - Parte do bolo alimentar preenchendo a valécula e parte ainda em cavidade oral.

3. Bolo alimentar preenchendo a valécula.

4. 3,5-Parte do bolo alimentar na valécula e parte escorrendo para marcar os seios piriformes.

5. Alimento já em seios piriformes.

Figura 2 - Diferentes posições do bolo alimentar no início da resposta faríngea.
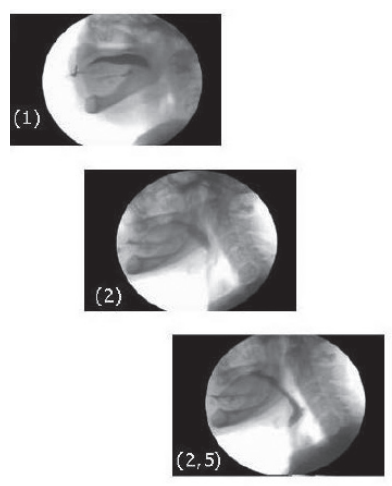

Fonte: Gatto (2010)

\section{Análise do Exame Videofluoroscópico}

Os exames, codificados para que os pesquisadores estivessem cegos aos dados clínicos dos voluntários, foram analisados por dois fonoaudiólogos. Em caso de divergência de resposta, um terceiro fonoaudiólogo foi consultado, considerando-se sua avaliação como critério de desempate.
A fase esofágica da deglutição foi avaliada pelo radiologista, considerando-se aspectos como presença de ondas terciárias, tempo de esvaziamento gástrico e presença de acalásia.

O grau de megaesôfago foi classificado conforme a descrição de Rezende (1997).

Grupo I - Esôfago de calibre aparentemente normal ao exame radiológico. Trânsito lento. Pequena retenção na radiografia tomada um minuto após a ingestão de sulfato de bário.

Grupo II - Esôfago com pequeno a moderado aumento do calibre. Apreciável retenção de contraste. Presença freqüente de ondas terciárias, associadas ou não a hipertonia do esôfago.

Grupo III - Esôfago com grande aumento de diâmetro. Atividade motora reduzida. Hipotonia do esôfago inferior. Grande retenção de contraste.

Grupo IV - Dolicomegaesôfago. Esôfago com grande capacidade de retenção, atônico, alongado, dobrando-se sobre a cúpula diafragmática.

A caracterização do grupo I está baseada nos seguintes aspectos: diâmetro esofagiano normal (menos de 3,2 $\mathrm{cm})$, retenção de bário no esôfago com nível perpendicular à parede esofagiana, presença de ar acima da coluna de bário associada a lúmen completamente aberto. Tais achados compõem a denominada "prova de retenção positiva", que pode identificar muitos casos da forma anectásica (sem dilatação) desta afecção.

\section{Análise Estatística}

Para elaboração e processamento do banco de dados foi utilizado o programa Statistical Package for Social Sciences (SPSS) for Windows versão 15 . Foi realizada a análise descritiva para caracterizar a distribuição dos eventos estudados. Para as variáveis categóricas foram encontradas as frequências absolutas simples e para as variáveis contínuas as medidas de tendências central (média, moda e mediana) e de dispersão (desvio-padrão). Proporções serão comparadas utilizando-se o teste qui-quadrado de 
Pearson ou teste de Fischer. A existência da associação entre as variáveis será considerada estatisticamente significativa a um nível descritivo ( $p$-valor) inferior a $5 \%$.

\section{RESULTADOS}

No total 26 participantes foram incluídos no estudo. A maior prevalência de indivíduos com diagnóstico de megaesôfago foi no sexo feminino, $73,1 \%(N=19)$ e o número de adultos jovens foi igual ao de idosos (Tabela 1)

Tabela 1 - Distribuição por faixa etária dos pacientes com megaesôfago chagásico, média, mediana, valores mínimo e máximo.

\begin{tabular}{lrc}
\hline \multicolumn{1}{c}{ Faixa Etária } & \multicolumn{1}{c}{ Valores } & N \\
\hline$<60$ anos & $50 \%$ & 13 \\
$\geq 60$ anos & $50 \%$ & 13 \\
Média & 59,20 anos & - \\
Mediana & 60 anos & - \\
Valor mínimo & 35 anos & - \\
Valor máximo & 77 anos & - \\
\hline
\end{tabular}

Fonte: Autoria própria

A amostra estudada apresenta graus iniciais de megaesôfago, segundo a classificação de Rezende (1997), grau | 30,4\% e grau || 39,1\% (Tabela 2). Nesta variável houve perda de 3 indivíduos com diagnóstico confirmado de megaesôfago, por impossibilidade de análise do exame para definição do grau.

Tabela 2 - Distribuição do grau de megaesôfago conforme Rezende (1997)

\begin{tabular}{lcc}
\hline Grau do Megaesôfago & Percentual & N \\
\hline Grau I & $30,5 \%$ & 7 \\
Grau II & $39,1 \%$ & 9 \\
Grau III & $17,4 \%$ & 4 \\
Grau IV & $13,0 \%$ & 3 \\
\hline
\end{tabular}

Fonte: Autoria própria

Com relação ao número de cirurgias realizadas para abertura da cárdia, um percentual de $50 \%$ nunca realizou e $34,6 \%$ realizou uma abordagem cirúrgica (Tabela 3 ).

Tabela 3 - Frequência de cirurgias realizadas para abertura da cárdia.

\begin{tabular}{lcc}
\hline Número de cirurgias & Percentual & N \\
\hline Nenhuma & $50 \%$ & 13 \\
Uma & $34,6 \%$ & 9 \\
Duas & $15,4 \%$ & 4 \\
\hline
\end{tabular}

Fonte: Autoria própria

O tempo de trânsito oral total está demonstrado na figura 3. O sólido, pela necessidade de preparo, apresenta um tempo muito maior. As demais consistências apresentaram tempo de trânsito oral total menor que 10 segundos. O tempo do líquido não foi calculado, devido à dificuldade em analisar as fases inicial e final.

Figura 3 - Boxplot referente ao tempo de trânsito oral nas diferentes consistências.

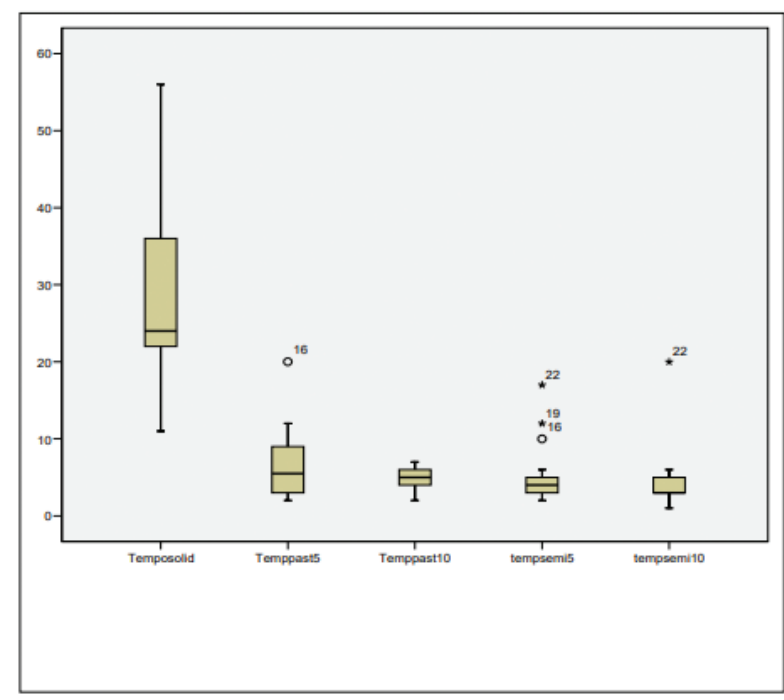

Fonte: Autoria própria

A Figura 4 demonstra que o tempo de trânsito oral de sólidos foi maior nos participantes com ausência de dentes posteriores inferiores e superiores inferiores.

Figura 4 - Boxplot referente ao tempo de trânsito oral de sólidos em diferentes conformações dentárias

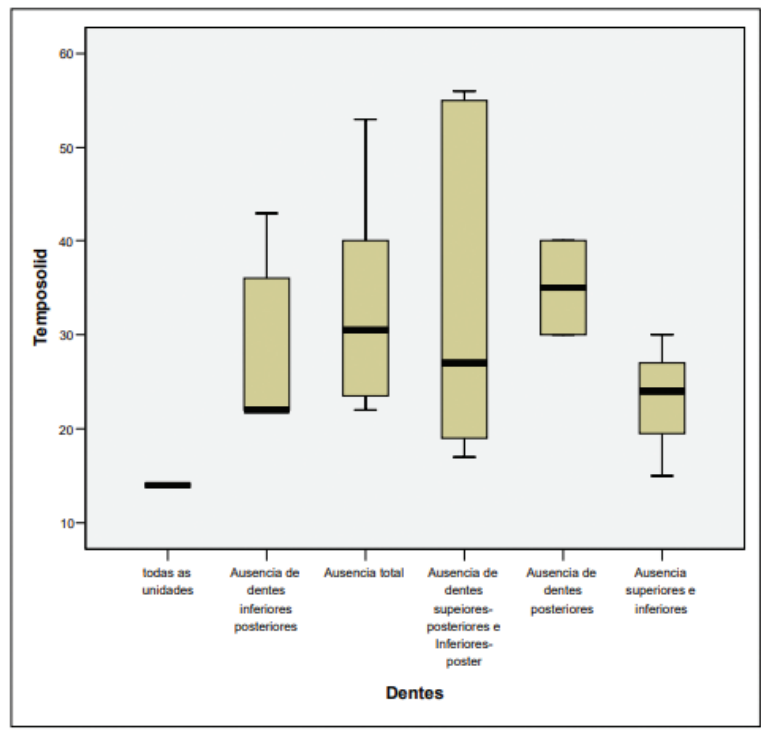

Fonte: Autoria própria

A ausência de peças dentárias foi predominante na amostra, principalmente de dentes posteriores, somando 90,90\%. Dentre os pacientes, 33,3\% apresentaram ausên- 
cia total de dentes, $28,8 \%$ ausência de dentes posteriores inferiores e $28,8 \%$ ausência de dentes posteriores inferiores e superiores. A mastigação anterior ocorreu em $40,7 \%$.

Resíduo oral após deglutição foi observado em $14,8 \%$ ao deglutir sólido, $11,1 \%$ pastoso, $77,8 \%$ para $5 \mathrm{ml}$ de semilíquido, $88,9 \%$ para $10 \mathrm{ml}$ de semilíquido e $74,1 \%$ para líquido. A presença de resíduo oral na consistência semilíquida associou-se, de forma significativa, à presença de resíduo oral de líquido. $(P=0,012)$.

Um percentual de $83,3 \%$ dos pacientes com presença de resíduo oral para $10 \mathrm{ml}$ de semilíquido também apresentaram resíduo na consistência líquida.

A tabela 4 representa os achados da posição do bolo alimentar no início da resposta faríngea, segundo classificação de Gatto (2010).

Tabela 4 - Achados da posição do bolo alimentar no início da resposta faríngea segundo Gatto (2010).

\begin{tabular}{|c|c|c|c|c|c|c|c|c|c|c|c|c|}
\hline \multirow[t]{2}{*}{ Classificação } & \multicolumn{2}{|c|}{ Sólido } & \multicolumn{2}{|c|}{ Pastoso $5 \mathrm{ml}$} & \multicolumn{2}{|c|}{ Pastoso $10 \mathrm{ml}$} & \multicolumn{2}{|c|}{ Semi líquido $5 \mathrm{ml}$} & \multicolumn{2}{|c|}{$\begin{array}{c}\text { Semi líquido } \\
10 \mathrm{ml}\end{array}$} & \multicolumn{2}{|c|}{ Líquido } \\
\hline & $\%$ & $\mathrm{~N}$ & $\%$ & $N$ & $\%$ & $\mathrm{~N}$ & $\%$ & $\mathrm{~N}$ & $\%$ & $N$ & $\%$ & $\mathrm{~N}$ \\
\hline 1 & 11,1 & 3 & 40,7 & 11 & 44,4 & 12 & 40,7 & 11 & 44,4 & 12 & 48,1 & 13 \\
\hline 2 & 7,4 & 2 & 18,5 & 5 & 29,5 & 7 & 0 & 0 & 0 & 0 & 3,7 & 1 \\
\hline 2,5 & 38,5 & 10 & 14,8 & 4 & 25,9 & 7 & 11,1 & 3 & 7,4 & 2 & 11,1 & 3 \\
\hline 3 & 19,2 & 5 & 14,8 & 4 & 7,4 & 2 & 22,2 & 6 & 18,5 & 5 & 11,1 & 3 \\
\hline 3,5 & 7,4 & 2 & 11,1 & 3 & 7,4 & 2 & 7,4 & 2 & 11 & 3 & 7,4 & 2 \\
\hline 4 & 15,4 & 4 & 0 & 0 & 3,7 & 1 & 18,5 & 5 & 18,5 & 5 & 18,5 & 5 \\
\hline
\end{tabular}

Classificação segundo Gatto (2010) - 1. Bolo alimentar em qualquer região entre pilar das fauces e o ponto onde o dorso da língua cruza a borda inferior da mandíbula; 2 . Bolo alimentar no dorso da língua quase atingindo a valécula; 2,5 . Parte do bolo alimentar preenchendo a valécula e parte ainda em cavidade oral; 3. Bolo alimentar preenchendo a valécula; 3,5. Parte do bolo alimentar na valécula e parte escorrendo para marcar os seios piriformes; 4 . Alimento já em seios piriformes.

Fonte: Autoria própria

O escape posterior mais alterado foi observado para alimentos sólidos $(15,4 \%)$ e em seguida para semilíquido $10 \mathrm{ml}$. As demais consistências apresentaram a maior parte do seu percentual no limiar de normalidade.

O clearance faríngeo foi mais observado no alimento sólido (100\%) e pastoso $5 \mathrm{ml}(96,3 \%)$ e $10 \mathrm{ml}(92,6 \%)$. Nas demais consistências a presença de resíduo faríngeo foi observada, $51,9 \%$ para $5 \mathrm{ml}$ de semilíquido, $70,4 \%$ para $10 \mathrm{ml}$ de semilíquido e $63 \%$ para o líquido.

As consistências de maior retenção na região de transição faringoesofágica foram as semilíquida $10 \mathrm{ml}$ $(85,2 \%)$, líquida $(77,8 \%)$ e semilíquida $5 \mathrm{ml}(70,4)$.

Um percentual muito pequeno $-3,7 \%$ - de penetração laríngea foi encontrado ao deglutir semilíquido e líquido. 0 mesmo percentual apresentou aspiração laringotraqueal ao deglutir $10 \mathrm{ml}$ de semilíquido.

Nenhuma associação estatisticamente significativa foi observada entre os achados videofluoroscópicos e o grau de megaesôfago.

\section{DISCUSSÃO}

Neste estudo encontramos ausência de unidades dentárias na maioria dos voluntários, tempo de trânsito oral aumentado em todas as consistências, principalmente para sólidos. O resíduo oral foi mais encontrado ao se deglutir líquidos e semilíquidos, assim como o resíduo faríngeo. Nessas consistências, a presença do alimento na transição faringoesofágica foi registrada.

Outros estudos demonstram a incidência de doença de Chagas em mulheres adultas e pesquisas com essa população têm destacado a necessidade de prevenir a doença de Chagas congênita, a partir de triagens e ações de saúde pública voltadas para a identificação e tratamento precoce (MONGE-MAILLO; LÓPEZ-VÉLEZ, 2017).

Metade da população foi de indivíduos idosos, os quais, além das alterações fisiológicas causadas pelo megaesôfago, tendem a apresentar modificações estruturais anatômicas que modificam o processo de deglutição, apesar do impacto da idade não ter sido descrito em estudo que comparou a dinâmica de deglutição de líquidos entre indivíduos idosos e jovens portadores de acalásia (DANTAS et al., 2018).

A disfagia secundária ao comprometimento esofágico dos indivíduos portadores de doença de Chagas é descrita como um sintoma que piora a qualidade de vida e leva a complicações, como desnutrição e pneumonias aspirativas, ocorrendo em $8 \%$ a $40 \%$ dos casos (MURARO; CAMARGO; AQUINO, 2009). Neste sentido, tratar essa condição é importante para reduzir complicações e a cirurgia tem se mostrado efetiva nos casos mais leves, como na maioria dos casos deste estudo. Uma limitação neste tópico foi a descrição do tipo de cirurgia, visto que na literatura muitas são as referências para esse procedimento (PANDOLFINO; GAWRON, 2015).

O significativo percentual da perda de peças dentárias na população estudada pode ser justificado por ser a doença de Chagas mais incidente em populações de baixo nível sócio-econômico e de menor escolaridade (BERNABÉ; MARCENES, 2011; PERES et al., 2013). A pesquisa nacional de saúde bucal, realizada em 2010, descreve ainda que a Região Nordeste apresenta indivíduos com maior perda dentária funcional quando comparada a outras regiões do país (PERES et al., 2013), 
sendo este mais um fator que corrobora com os resultados deste estudo.

A ausência de peças dentárias causa problemas funcionais, tais como dificuldades em mastigar e falar, dor, além de transtornos sociais, como insatisfação com a aparência e dificuldade no convívio (BITENCOURT; CORRÊA; TOASSI, 2019; JORGE et al., 2009).

$\mathrm{Na}$ fase preparatória da deglutição os dentes desempenham um papel muito importante, degradando os alimentos sólidos para a formação do bolo alimentar (COSTA, 1998). Em situações de redução de peças dentárias, adaptações como mudanças na consistência do alimento e a manipulação lenta do bolo alimentar são descritas, gerando aumento do tempo de trânsito oral, conforme encontrado nos resultados deste estudo (VENITES; SOARES; BILTON, 2018).

Os tempos de trânsito oral para pastoso e semilíquido na população estudada encontram-se aumentados quando comparados aos controles saudáveis de alguns estudos (GOMES et al., 2008; SANTOS; CASSIANI; DANTAS, 2011; SILVA; DANTAS; FABIO, 2010) e a indivíduos com doença de Chagas (GOMES et al., 2008; SANTOS; CASSIANI; DANTAS, 2011). Nessas pesquisas os tempos de trânsito orais para pastosos e semilíquidos foram menores que 1 segundo.

A presença de resíduo oral também foi descrita em pacientes com doença de Chagas ao deglutirem alimento pastoso no estudo de Gomes et al. (2008), divergindo dos achados de Santos, Cassiani e Dantas ( 2011).

O escape precoce foi observado principalmente nas consistências sólida e semilíquida, na sua maior oferta. Alguns estudos observam a influência da mastigação na preparação do bolo alimentar e no início da deglutição, sugerindo que a mastigação parece reduzir a efetividade do selamento posterior língua-palato, permitindo o escape do conteúdo oral para a faringe (SAITOH et al., 2007; SALIBA et al., 2010). Já o escape presente na consistência semilíquida pode ser causado pela falta de controle na contenção desse alimento na boca, seja decorrente do volume ou da menor densidade (QUEIROZ; HAGUETTE; HAGUETTE, 2009).

A presença de resíduos alimentares em valéculas e/ ou recessos piriformes pode ocorrer devido às alterações na fase preparatória e/ou oral da deglutição, à ineficiência da ejeção do bolo alimentar, ao atraso no disparo do reflexo da deglutição, à diminuição dos movimentos peristálticos, à redução da elevação e à anteriorização laríngea e/ou à incoordenação do músculo cricofaríngeo (COSTA et al., 1993).

No nosso estudo, o semilíquido e líquido foram os alimentos que mais apresentaram resíduos orais, faríngeos e em região de transição faringoesofágica. Já o estudo realizado por Gomes et al. (2008), apresentou resíduo em todas as regiões citadas, porém na consistência pastosa, que tem maior viscosidade que a semilíquida.

A ausência de peças dentárias e conseqüente déficit de força muscular orofacial podem ter favorecido o déficit na fase oral da deglutição, o que contribuiu para a presen- ça de resíduos orais, faríngeos e em região de transição faringoesofágica. A presença de resíduo na consistência semilíquida talvez tenha ocorrido pela diminuída força ejetora, conseqüência da reduzida propriocepção oral dessa consistência, o que não deve ter ocorrido no pastoso por ter maior percepção oral devido a sua viscosidade.

Penetração laríngea e aspiração ocorreram no mesmo paciente, que também apresentou escape posterior igual a 4, resíduo faríngeo e em região de transição faringoesofágica após deglutições. $O$ escape posterior e a presença de aspiração podem ser justificados pela maior chance de aspirar devido à permanência da abertura da via aérea até que a deglutição seja deflagrada.

\section{CONCLUSÃO}

No grupo estudado, foram observados, na fase oral, maior tempo de trânsito, mastigação anterior e presença de resíduo oral nas consistências semilíquida e líquida, enquanto que, na fase faríngea, observou-se a presença de resíduo faríngeo e retenção em transição faringoesofágica também nas consistências semilíquida e líquida. A presença de penetração e aspiração foram observadas em apenas um caso.

\section{REFERÊNCIAS}

BERNABÉ, E. ; MARCENES, W. Income inequality and tooth loss in the United States. J. dent. res., Washington, v. 90, n.6, p. 724729, 2011. Disponível em: http://journals.sagepub.com/doi/ abs/10.1177/0022034511400081. Acesso em: 10 oct. 2019.

BITENCOURT, F. V.; CORRÊA, H. W.; TOASSI, R. F. C. Experiências de perda dentária em usuários adultos e idosos da Atenção Primária à Saúde. Ciênc. saúde colet., Rio de Janeiro, v. 24, p. 169-180, 2019. Disponível em: https://www.scielosp.org/pdf/csc/2019.v24n1/169-180. Acesso em: 10 out. 2019.

BRASIL. Ministério da Saúde. Agência Fiocruz de notícias. Doença de chagas. Brasilia: MS, 10 set. 2013. Disponível em: https://agencia.fiocruz. br/doen\%C3\%A7a-de-chagas. Acesso em: 28 abr. 2019.

BUENO, M. M. S.; ALVES, O. de F. Manifestações da Doença de Chagas no esôfago. Saúde \& Ciência em Ação - Revista Acadêmica do Instituto de Ciências da Saúde, Goiânia, v. 1, n. 1, p.119-131, 2015. Disponível em: http://revistas.unifan.edu.br/index.php/RevistalCS/article/ view/109/90. Acesso em: 10 out. 2019.

COSTA, M. M. B. et al. A avaliação videofluoroscópica da transição faringoesofágica (esfíncter superior do esôfago). Radiol. bras., Rio de Janeiro, v. 26, n. 2, p. 71-80, 1993. Disponível em: http://bases.bireme. $\mathrm{br} / \mathrm{cgi}$-bin/wxislind.exe/iah/online/?lsisScript=iah/iah.xis\&src=google\& base=ADOLEC\&lang=p\&nextAction=Ink\&exprSearch=169823\&indexSe arch=ID. Acesso em: 10 out. 2019.

COSTA, M. M. B. Dinâmica da deglutição: fase oral e faríngea. In: COSTA, M. M. B.; LEME, E.; KOCH, H. I. (Eds.) Colóquio multidisciplinar deglutição e disfagia do Rio de Janeiro. 1998.

DANTAS, R. O. et al. Water ingestion dynamics in patients with achalasia: influence of sex and age. Arq. gastroenterol., São Paulo, v. 55, p 25-28, 2018. Disponível em: http://www.scielo.br/pdf/ag/v55s1/1678-4219-ag-s0004280320180000043.pdf. Acesso em: 10 out. 2019.

DIAS, J. C. P. et al. II Consenso Brasileiro em Doença de Chagas, 2015. Epidemiol. serv. saúde, Brasília, v. 25, n. 21, p.7-86, 2016. Instituto Evandro 
Chagas.Disponível em: https://www.scielosp.org/pdf/ress/2016.v25nspe./7-86. Acesso em: 10 out. 2019.

GATTO, A. R. Efeito do sabor azedo e da temperatura fria na fase oral da deglutição no acidente vascular encefálico. 2010. 91f. Dissertação (Mestrado em Bases Gerais da Cirurgia) — Universidade Estadual Paulista Júlio de Mesquita Filho, Botucatu 2010. Disponível em: https://repositorio.unesp.br/bitstream/handle/11449/86302/gatto_ar_me_botfm. pdf?sequence=1. Acesso em: 10 out. 2019.

GOMES, F. R. et al. Oral and pharyngeal transit of a paste bolus in chagas' disease. Dysphagia, New York, v. 23, p. 82-87, 2008. Disponível em: https://link.springer.com/article/10.1007/s00455-007-9101-8. Acesso em: 10 out. 2019.

GONÇALVES, M. I. R.; LEDERMAN, H. M; Protocolo de Avaliação videofluoroscópica de deglutição de adultos - Video de glutoesofagograma. Fono atual, São Paulo, v. 27, n. 27, p 78-86, 2004.

JORGE, T. M. et al. Relação entre perdas dentárias e queixas de mastigação, deglutição e fala em indivíduos adultos. Revista CEFAC, São Paulo, v.11, p. 391-397, 2009. Disponível em: https://www.redalyc.org/ pdf/1693/169318767014.pdf. Acesso em: 10 out. 2019.

MONGE-MAILLO, B.; LÓPEZ-VÉLEZ, R. Challenges in the Management of chagas disease in Latin-American migrants in Europe. Clin. microbiol. infect., Oxford, v. 23, p. 290-295, 2017. Disponível em: https://reader. elsevier.com/reader/sd/pii/S1198743X17302240?token=FE6C1E8469 BC5F25516D85CCFED08B8FF3F4588649A597B73F756634173CBCE7 B5DD9B3D2254AA5826950F1C48890A0A. Acesso em: 10 out. 2019.

MURARO, C. P. M.; CAMARGO, J.G.T.; AQUINO, J.L.B. Megaesôfago. MURARO, C.P.M. Cirurgia do aparelho digestório. Rio de Janeiro: Editora Rubio, 2009. p. 19-27.

MURILLO-GODÍNEZ, G. Enfermedad de chagas (tripanosomiasis americana). Med. interna Méx.. México, v. 34, n. 6, p. 959-970, 2018.

PANDOLFINO, J. E.; GAWRON, A. J. Achalasia a systematic review. JAMA, Chicago, v. 313, n. 18, p 1841-1852, 2015. Disponível em: https:// jamanetwork.com/journals/jama/article-abstract/2290638. Acesso em: 10 out. 2019.

PERES, M. A. et al. Perdas dentárias no Brasil: Análise da Pesquisa Nacional de Saúde Bucal 2010. Rev. Saúde Pública, São Paulo, v. 47, n. 3, p 78-89, 2013. Disponível em: https://www.scielosp.org/pdf/rsp/2013. v47suppl3/78-89. Acesso em: 10 out. 2019.

QUEIROZ, M. A. S.; HAGUETTE, R. C. B.; HAGUETTE, E. F. Achados da videoendoscopia da deglutição em adultos com disfagia orofaríngea neurogênica. Rev. Soc. Bras. Fonoaudiol., São Paulo, v. 14, n. 3, p 454462, 2009.

REZENDE, J. M. O Aparelho Digestivo na Doença de Chagas - Aspectos Clínicos. In: DIAS, J. C. P.; COURA, J. R. (Eds.). Clínica e terapêutica da doença de Chagas: uma abordagem prática para o clínico geral. Rio de Janeiro: Fiocruz, 1997. p. 154-176.

SAITOH, E. et al. Chewing and food consistency: effects on bolus transport and swallow initiation. Dysphagia, New York, v. 22, p.100-107, 2007. Disponivel em: https://link.springer.com/article/10.1007/s00455-0069060-5. Acesso em: 10 out. 2019.

SALIBA, N. A. et al. Perda dentária em uma população rural e as metas estabelecidas pela Organização Mundial de Saúde. Ciênc. Saúde Colet., Rio de Janeiro, v. 15, n .1, p 1857-1864, 2010. Disponível em: https:// www.scielosp.org/pdf/csc/2010.v15suppl1/1857-1864. Acesso em: 10 out. 2019.

SANGENIS, L. H. C. et al. Transmissão da doença de Chagas por consumo de carne de caça: revisão sistemática. Rev. bras. epidemiol., São Paulo, v. 19, n.4, p 803-811, 2016. Disponível em: https://www.scielosp.org/ pdf/rbepid/2016.v19n4/803-811. Acesso em: 10 out. 2019.

SANTOS, C. M. dos; CASSIANI, R. de A.; DANTAS, R. O. Alterações da deglutição na doença de Chagas. Rev. Bras. Clín. Méd., São Paulo, v. 10, n. 3, p. 219-221, 2012. Disponível em: http://www.sbcm.org.br/ revistas/RBCM/RBCM-2012-03. pdf\#page=46. Acesso em: 10 out. 2019.

SANTOS, C. M. dos; CASSIANI, R. de A.; DANTAS, R. O. Videofluoroscopic Evaluation of Swallowing in Chagas' Disease. Dysphagia, New York, v. 26, p 361-365, 2011. Disponível em: https://link.springer.com/article/10.1007/s00455-010-9321-1. Acesso em: 10 out. 2019.

SANTOS, F. da S. dos et al. Doença de Chagas e sua transmissão pelo açaí: Uma revisão bibliográfica. Braz. J. health rev., [s.I], v. 2, n. 3, p. 2128-2144, 2019. Disponível em: http://brjd.com.br/index.php/BJHR/ article/view/1595. Acesso em: 10 out. 2019.

SILVA, A. C. V. da; DANTAS, R. O.; FABIO, S. R. C. Clinical and scintigraphic swallowing evaluation of post-stroke patients. Pró-Fono R. Atual. Cient., Barueri, v.22, n. 3, p. 215-220, 2010. Disponível em: http://www.scielo. br/pdf/pfono/v22n3/en_a27v22n3.pdf. Acesso em: 10 out. 2019.

VENITES, J.; SOARES, J.; BILTON, T. (Orgs.) Presbifagia, disfagia no idoso e disfagia sarcopênica. In: SOARES, J.; VENITES, J. P.; SUZUKI, H. Disfagia no Idoso. Ribeirão Preto: Booktoy, 2018. p. 81-86.
Submetido em: 04/11/2019

Aceito em: 30/11/2019 